\title{
Politique
}

Politique

\section{Korany Bahgat (sous la direction de), « La Crise des relations \\ internationales : vers un bilan ", Études internationales, vol. XV n ${ }^{0} 4$, décembre 1984.}

\section{Diane Ethier}

Numéro 8, automne 1985

Innovations et politiques technologiques

URI : https://id.erudit.org/iderudit/040512ar

DOI : https://doi.org/10.7202/040512ar

Aller au sommaire du numéro

Éditeur(s)

Société québécoise de science politique

ISSN

0711-608X (imprimé)

1918-6584 (numérique)

Découvrir la revue

Citer ce compte rendu

Ethier, D. (1985). Compte rendu de [Korany Bahgat (sous la direction de), « La

Crise des relations internationales : vers un bilan ", Études internationales,

vol. XV ${ }^{0}$ 4, décembre 1984.] Politique, (8), 180-183.

https://doi.org/10.7202/040512ar

Ce document est protégé par la loi sur le droit d'auteur. L'utilisation des services d'Érudit (y compris la reproduction) est assujettie à sa politique d'utilisation que vous pouvez consulter en ligne.

https://apropos.erudit.org/fr/usagers/politique-dutilisation/ 
Korany Bahgat (sous la direction de), «La Crise des relations internationales: vers un bilan, Études internationales, vol. XV no 4, décembre 1984.

Réalisé sous la direction de Bahgat Korany, ce numéro spécial d'Études internationales nous propose une synthèse critique de l'évolution des théories des relations internationales. Comprenant neuf articles, rédigés par des spécialistes de la discipline, l'ouvrage se présente comme un bilan des méthodes et des débats qui ont caractérisé, depuis trente ans, les principaux domaines d'études de ce sous-champ de la science politique. Empruntant une démarche rétrospective, critique et prospective, les auteurs cherchent à cerner les fondements, les contradictions, les limites et les conditions de renouvellement des divers paradigmes de cette branche de la connaissance.

Bien que cette publication ne soit pas la première à offrir, en français, un tour d'horizon des théories des relations internationales (voir P. Braillard (1977), J.P. Gonidec (1974), M. Merle (1974), C. Zorghibe (1975)), elle deviendra sans doute un des instruments de référence privilégié sur le sujet, non seulement parce qu'elle contient un inventaire bibliographique des sources fondamentales aussi riche que celui de ces manuels tout en étant plus succincte, mais parce qu'elle pose une série de questions novatrices sur l'objet d'étude et les paramètres méthodologiques de la discipline.

L'avant-propos et les deux premiers articles du numéro, signés par Bahgat Korany, soulèvent en effet, à l'instar de d'autres revues spécialisées (Theory and Decision (1982), International Organization (1984)) la question de la crise épistémologique des relations internationales. Cette crise, selon l'auteur, se manifeste par l'absence de diagnostics et d'hypothèses prospectives convergentes sur les transformations du système mondial. En raison, d'une part, de la complexité croissante du système, et, d'autre 
part, de l'hétérogénéité des modèles théoriques et des conceptions idéologiques défendues au sein de la discipline, il n'existe pas de perception consensuelle et universelle de l'ordre international. Cet état de choses suggère une remise en cause du statut scientifique de la discipline et de la capacité des spécialistes à guider l'action des intervenants/acteurs (États, organisations internationales, forces transnationales). Il convient donc de s'interroger, non seulement sur les fondements et les lacunes épistémologiques de chaque école de pensée, mais sur les possibilités d'intégration et de renouvellement des instruments conceptuels utilisés.

Ces propositions, comme le constateront les lecteurs du volume, ne sont pas entérinées par tous les chercheurs. Dans son article consacré à la critique et au survol de la documentation des études stratégiques, Albert Legault défend un point de vue opposé.

"Il n'y a pas à proprement parler de crise dans les relations internationales, pas plus qu'il n'y a de crise dans les études stratégiques. Aucune théorie globalisante ne saurait expliquer l'évolution des rapports entre États, pas plus qu'une théorie spécifique ne saurait expliquer la réalité. Chaque théorie, chaque discipline, chaque paradigme ne vaut que par rapport à l'objet social découpé. Or, celui-ci refuse de se laisser enfermer dans un cadre trop étroit». (p. 747) Michael Brecher et J. David Singer rejettent également l'hypothèse d'une crise épistémologique des relations internationales, cherchant à démontrer dans leurs études synthèses, la validité et le dynamisme des modèles systémique (Brecher) et behavioriste (Singer).

Paul N. Dussault, dont la réflexion porte sur les origines et l'évolution du concept d'acteur et A. Donneur et O. Beylerian, qui tracent un portrait minutieux de l'état des travaux en politique étrangère, adoptent quant à eux une position plus critique. Tout en reconnaissant l'apport scientifique indéniable des théories sur le comportement et la prise de décision des acteurs politiques individuels et institutionnels, théories dont le principal avantage 
a été de dépasser le déterminisme trop rigide des interprétations systémiques, ils soulignent l'importance des nouvelles approches fournies par l'économie politique. P. N. Dussault mentionne à cet égard l'intérêt des analyses sur la multinationalisation des firmes (C. A. Michalet, C. Palloix) tandis que A. Donneur et O. Beylerian insistent longuement sur les possibilités méthodologiques offertes par la nouvelle approche d'Immanuel Wallerstein sur l'économie-monde capitaliste. Cette section du texte de A. Donneur et $\mathrm{O}$. Beylerian est particulièrement intéressante puisqu'elle complète l'exposé de Wallerstein sur «Les Tendances et les perspectives de l'économie-monde».

L'article de I. Wallerstein occupe une place privilégiée au sein de l'ouvrage puisqu'il se situe au-delà du débat actuel en proposant une nouvelle alternative théorique. Offrant une définition plus rigoureuse et plus cohérente du concept d'économie-monde capitaliste que les écrits antérieurs de l'auteur, il constitue une excellente base d'évaluation de cette nouvelle vision structuraliste, multidisciplinaire et globalisante de l'ordre capitaliste mondial.

En définitive, comme l'indique la conclusion de Bahgat Korany sur «l'au-delà de la crise», les différents articles de ce numéro reflètent très bien l'état des positions actuelles sur le statut épistémologique des relations internationales. Alors que plusieurs chercheurs associent encore prioritairement cette discipline à l'étude des relations inter-étatiques diplomatiques et stratégiques, privilégiant les approches post-réalistes et néo-positivistes américaines, d'autres, moins nombreux, prônent l'intégration de l'objet et des théories du développement et de l'économie politique au champ des relations internationales.

Au-delà de ces oppositions toutefois se dessinent certains consensus prometteurs: 1) «le concept de «système» n'est plus un «cri de guerre», mais c'est son application qui est en cause»; 2) on accepte que le système se compose de deux types d'acteurs internationaux (les États et les forces transnationales); 3) l'histoire 
et l'économie politique sont désormais reconnues comme disciplines nécessaires, la réhabilitation de cette dernière étant liée à l'abolition de la dichotomie simpliste entre «high politics» (politiques diplomatiques et stratégiques des États) et «low politics» (politiques économiques et sociales); 4) la division du travail mécaniste entre empiristes et rationalistes est remise en question de même que la vision naïve de la neutralité ou de l'objectivité des paradigmes de recherche.

Tant pour les non-initiés qui désirent débrouiller l'écheveau complexe des théories des relations internationales, que pour les initiés qui souhaitent connaître l'état du débat épistémologique au sein de la discipline, l'ouvrage offre un grand intérêt. Deux réserves doivent malgré tout être formulées: d'une part, les études sur les organisations internationales, les forces transnationales et les problèmes du développement sont plus ou moins exclues du bilan; d'autre part, le titre du numéro prête à confusion laissant entendre qu'il s'agit d'une réflexion sur la crise des relations internationales plutôt que sur la crise des théories des relations internationales.

Diane Ethier

Université de Montréal. 